\title{
Telemedizin im kassenärztlichen Bereitschaftsdienst mit Notfallsanitätern
}

Daniel Overheu

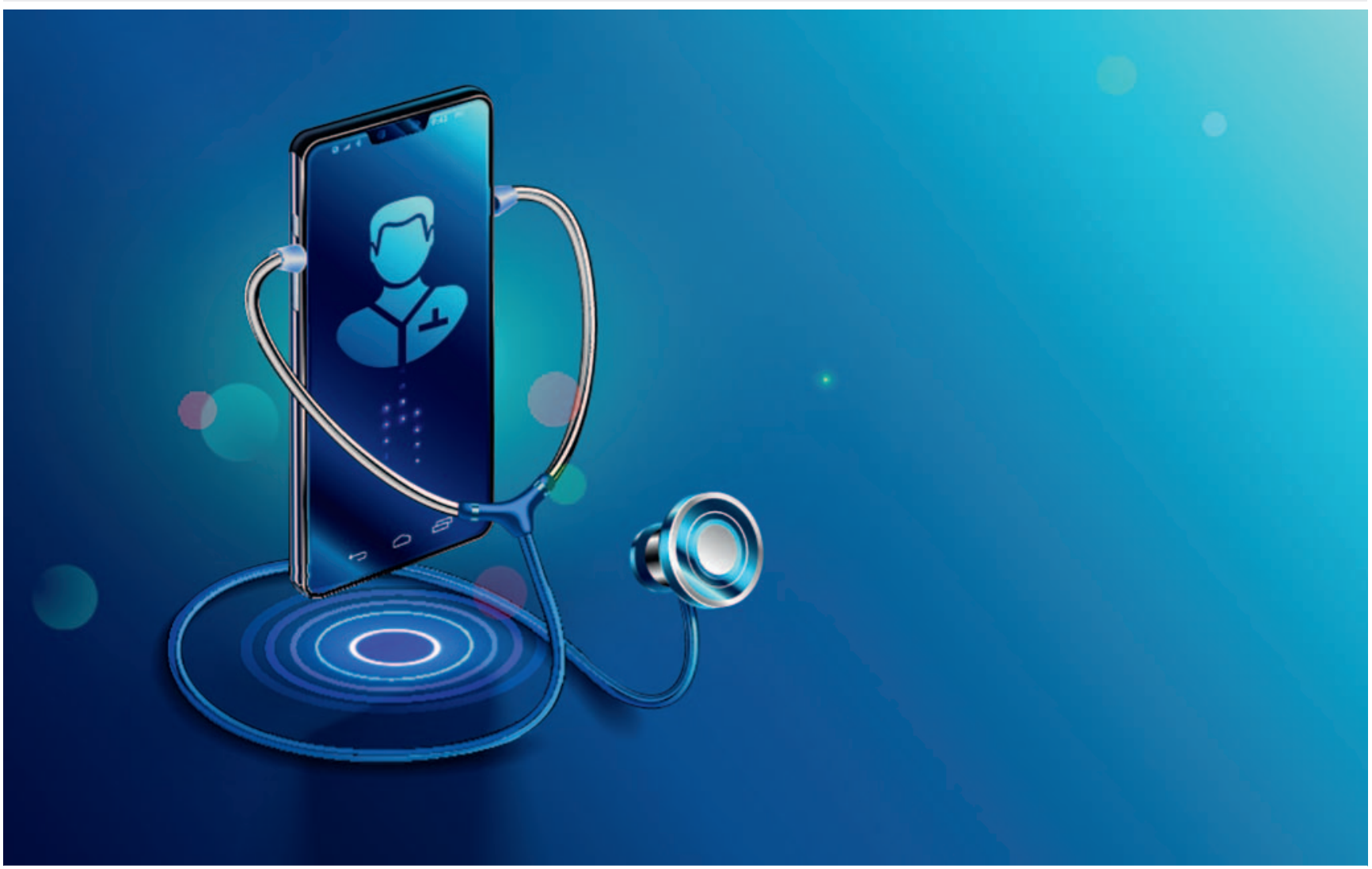

Quelle: AndSus/stock.adobe.com (Symbolbild)

Immer mehr alte Menschen und gleichzeitig immer weniger Ärztinnen und Ärzte - das stellt die Versorgung vor große Probleme. Technische Neuerungen wie die Telemedizin können helfen, die Versorgung aufrechtzuerhalten. Ein Modellprojekt in Niedersachsen testet neue Wege.

Die Bevölkerung in Deutschland wird immer älter. Für den Zeitraum bis 2060 wird ein Anstieg des Medianalters von 45 Jahren auf ca. 50 Jahre prognostiziert [1]. Weil die Gesundheitsleistungen für ältere Menschen erheblich über denen für jüngere liegen, wird ein steigender Bedarf an Gesundheitsleistungen vorhergesagt [2].

\section{Versorgungsengpässe im ambulanten Bereich}

Laut Ärztestatistik 2016 [3] steigt die Zahl der Ärzte/-innen im Akutsektor um 2,5\% und im ambulanten Sektor um 1,3\% - und damit langsamer als der Bedarf. Gleich- zeitig stagniert die Zahl der Studienanfänger/-innen bei rund 9500 Studierenden pro Jahrgang [4]. Ändert sich dies nicht, verschärft sich der Trend noch. Insbesondere bei Hausärzten/-innen ist ein Versorgungsengpass zu erwarten [5].

Versorgungsengpässe bei niedergelassenen Ärzten/-innen sind in erster Linie in Regionen zu erwarten, die als Wohnort und/oder hinsichtlich der Verdienstaussichten unattraktiv sind, und bei Hausärzten/-innen bereits zu beobachten. Betroffen sind in erster Linie ländliche Gebiete, aber auch Städte mit geringer Steuereinnahmekraft und ungünstiger Demografie [6]. 
Um dem sich andeutenden Versorgungsengpass in den Problembezirken entgegenzuwirken, hat die Kassenärztliche Vereinigung Niedersachsen (KVN) Maßnahmen wie Niederlassungsprämien und Umsatzgarantien für Hausärzte/-innen ergriffen. Diese allein sind jedoch nicht ausreichend. Insbesondere der Bereitschaftsdienst der niedergelassenen Fachärzte/-innen wirft Probleme auf. Die Versorgungsqualität variiert stark, und nicht selten werden Patienten/-innen durch den Rettungsdienst versorgt, obwohl sie von ihrer Krankheitsschwere her eher eine Versorgung durch den Bereitschaftsdienst benötigt hätten.

\section{Modellprojekt 116117}

Im Oldenburger Land haben sich die Kassenärztliche Vereinigung (KVN) und die Johanniter-Unfall-Hilfe Regionalverband Weser-Ems unter Projektleitung des Klinikums Oldenburg (KOL) des Problems angenommen. Der Bereitschaftsdienstbezirk Delmenhorst wurde als Modellregion gewählt, weil dort sowohl ländliche als auch städtische Gebiete zu versorgen sind. Delmenhorst ist im landesweiten Vergleich extrem finanzschwach und stellt einen sozialen Brennpunkt dar [7]. Lemwerder und Ganderkesee sind als typisch ländliche Gemeinden bereits deutlich vom demografischen Wandel betroffen [1]. Im Planungsbezirk Delmenhorst besteht eine leicht unterdurchschnittliche Versorgung mit Hausärzten/-innen (96,2\%, 2016), die sich bis 2030 weiter verschlechtern wird $(88,6 \%)$ (vertrauliche Mitteilung der Kassenärztlichen Vereinigung Niedersachsen).

\section{KV-Dienst durch Gesundheits- fachkräfte}

Als innovativer Ansatz wird in diesem Projekt die nächtliche Fahrbereitschaft im Rahmen des kassenärztlichen Bereitschaftsdienstes (Tel. 116117) durch eine examinierte Gesundheitsfachkraft (Qualifikation Rettungsassistent/in/Notfallsanitäter/-in/exam. Gesundheits-/Krankenpfleger/-in) der Johanniter-Unfall-Hilfe (JUH) mit telemedizinischem Support aus dem KOL unter Vorschaltung einer Telefonzentrale (JUH) durchgeführt. Als Partner sind der Rettungsdienst Delmenhorst sowie die Großleitstelle Oldenburger Land und das ortsansässige Josef-Hospital Delmenhorst (JHD) in das Projekt mit eingebunden. In diesem Konstrukt ist es somit möglich, Hilfe suchenden Patienten/-innen außerhalb der Praxisöffnungszeiten von Hausärzten/-innen ein System zur Verfügung zu stellen, das ressourcenschonend ist und die Versorgungsqualität aufrechterhält.

Ziel ist, die Krankenhaus-Notaufnahme und den Rettungsdienst von einer übermäßigen Belastung durch
Selbsteinweisungen zu entlasten. So könnten die Ressourcen in Rettungsdienst und Krankenhaus wieder den echten Notfällen, die sie benötigen, zur Verfügung stehen und würden nicht für allgemeinärztliche Aufgaben gebunden.

In der praktischen Umsetzung wird die Dienstbereitschaft durch Notfallsanitäter/-innen im Fahrdienst sichergestellt von Freitag 17:00 Uhr bis Montag 07:00. Erhalten bleiben die Sprechstundenzeiten des/der Bereitschaftsarztes/-ärztin: freitags 17:00 - 21:00 und samstags/sonntags jeweils 8:00 - 12:00 und 16:00 - 20:00 Uhr in der Notdienstpraxis am JHD. So bleibt der freie Zugang der Patienten/-innen zur direkten ärztlichen Behandlung uneingeschränkt.

\section{Ablauf des Notrufs}

Wählt nun ein Patient oder eine Patientin in der Zeit von Freitag 17:00 Uhr bis Montag 07:00 die zentrale Rufnummer 116117 für den Bereitschaftsdienst der KV, wird sein/ihr Anruf zunächst an die Telefonzentrale der JUH weitergeleitet. Dort wird durch einen festgelegten Ablauf der Patientenfall geführt ( $\bullet$ Abb. 1).

Unterstützt durch interaktive PowerPoint-Folien, navigiert der Disponent bzw. die Disponentin durch die wichtigsten Stichworte und fragt Daten bei dem Anrufer bzw. der Anruferin ab. Als Ergebnis kommt er zu einer Entscheidung und Einstufung des/der Patienten/-in:

- vitaler Notfall Übergabe an die 112,

- kein medizinisches Anliegen keine direkten Maßnahmen, ggf. Hilfestellung,

- medizinisches Anliegen und mobil Versorgung in der Bereitschaftspraxis,

- medizinisches Anliegen und nicht mobil bzw. Wartezeit nicht tolerierbar Versorgung als Hausbesuch.

Soll ein Hausbesuch erfolgen, macht sich die Gesundheitsfachkraft der JUH mit dem Fahrzeug auf den Weg zum/zur Patienten/-in. Alle Einsatzdaten werden durch ein Navigationssystem mit Datenübertragung direkt zum Fahrdienst übertragen. Die ausrückende Fachkraft wird somit direkt zum/zur Patienten/-in gelotst. Rückfragen oder Absprachen zu den Besuchen erfolgen per Handy.

Eine Einbindung in den BOS-Funk des Rettungsdienstes wurde unterlassen, da der KV-Bereitschaftsdienst keine öffentliche Rettungsdienstaufgabe erfüllt. Dem wurde auch in der Bekleidung des Personals Rechnung getragen: Es finden sich keine Rettungsdiensthosen mit Reflexstreifen oder rot-orange Jacken, um der Bevölkerung keinen Rettungsdienst light zu suggerieren. 


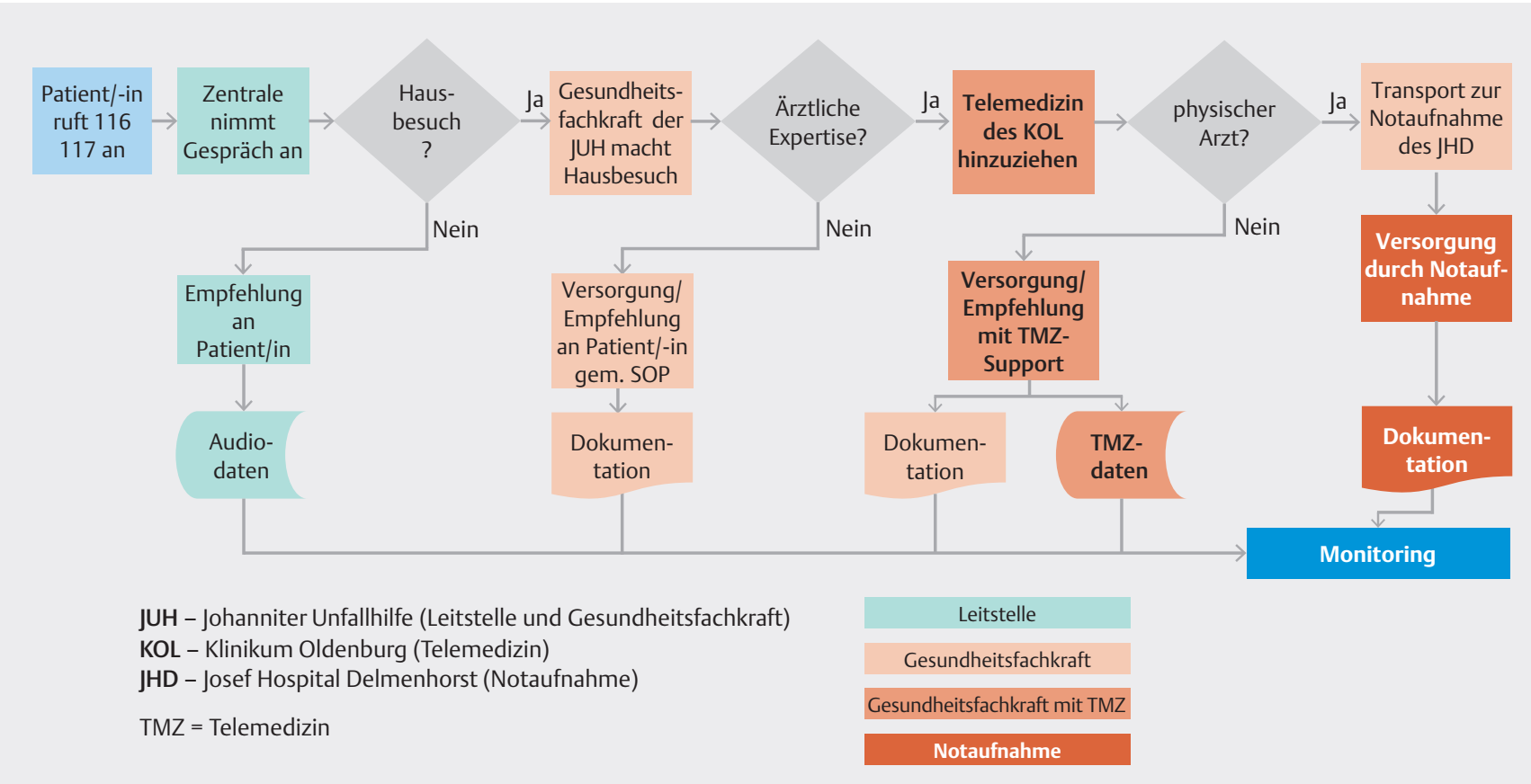

- Abb. 1 Ablaufschema Notruf KV-Bereitschaftsdienst.

\section{Training der Einsatzkräfte}

Bei den Patienten selbst steht der Gesundheitsfachkraft dann ein eigener Handlungsrahmen zur Verfügung.

Merke

In Schulungen wurden insgesamt 32 Standardprozeduren (SOPs) entwickelt und trainiert.

Darin fanden die vielfältigen Erfahrungen sowohl der JUH als auch des KOL Eingang, die beide gemeinsam schon seit 2015 mit telemedizinischer Unterstützung die Behandlung in der Offshore-Windindustrie in der Nordund Ostsee sicherstellen. Über 1500 Einsätze aus dem Offshore-Bereich wurden analysiert und die Erfahrungen zusammen mit einem langjährig erfahrenen KV-Bereitschaftsdienstarzt auf ihre Anwendbarkeit im Modellprojekt hin bewertet und in SOPs eingepflegt.

Jede SOP benennt auch klar die Differenzierung in eigenständig durchzuführende Maßnahmen, Maßnahmen in Rücksprache mit dem/der Telemediziner/-in und den Punkt der Abgabe des Falls an den örtlichen Rettungsdienst/Krankentransport. Neben SOPs wie Asthma bronchiale und Thoraxschmerz finden sich auch Vorgehensweisen für eher banale Themen wie entzündliche Prozesse, allgemeine Schmerztherapie oder akute Harnwegsinfekte. Jedes Teammitglied durchläuft vor dem Einsatz im Fahrdienst eine ärztlich supervidierte Schulung der SOPs, um die Anwendung der Maßnahmen beim Patienten/der Patientin lege artis sicher zu stellen.

\section{INFOBOX: NOTFALL-ALGORITHMEN}

Der Notfallsanitäter bzw. die Notfallsanitäterin im Einsatz hat alle lokalen Notfall-Algorithmen nach AG NUN zur Verfügung sowie folgende Spezialalgorithmen:

- Algorithmus Erregungszustand

- Algorithmus Analgesie

- Algorithmus Weichteilverletzung

- Algorithmus Wundversorgung

- Algorithmus Grippaler Infekt

- Algorithmus Halsschmerzen

- Algorithmus Kopfschmerzen

- Algorithmus Akuter Durchfall

- Algorithmus Blasenkatheter

- Algorithmus Harnwegsinfekt

\section{Einsatz von Telemedizin}

Zu jeder Zeit steht der Fachkraft vor Ort ein Telemediziner bzw. eine Telemedizinerin an der Universitätsklinik für Anästhesiologie am KOL zur Verfügung.

\section{Merke}

Über eine verschlüsselte Verbindung kann eine KV-zertifizierte Video-App gestartet werden, mit deren Hilfe eine Live-Videoverbindung zwischen Telemediziner/-in und Patient/-in hergestellt werden kann. 
Zusätzlich können ein 12-Kanal-EKG, die Pulsoxymetrie und mittels puls-transit-time noninvasive Blutdrücke übertragen werden. Der Telemediziner bzw. die Telemedizinerin kann sich also nicht nur ein klinisches Bild des/ der Patienten/-in verschaffen, sondern sich auf Vitalwerte stützen (Fallbeispiel).

\section{FALLBEISPIEL: MUSTEREINSATZ IM PROJEKT 116117}

An einem Samstagnachmittag geht über die Rufnummer 116117 ein Anruf ein. Der 37-jährige Alexander B. klagt über starke Rückenschmerzen. Seit 4 Tagen quält er sich, aber jetzt hilft scheinbar nichts mehr.

Als diensthabender Notfallsanitäter im Modellprojekt 116117 im Notdienstbezirk Delmenhorst übernimmt Johannes D. den Einsatz. Die Daten zum Patienten hat die Telefonzentrale der Johanniter-Unfall-Hilfe strukturiert abgefragt und auf sein Navigationsgerät übertragen. Dabei wurde auch geklärt, dass kein Notfall im Sinne des Rettungsdienstes vorliegt. Im Wohnzimmer von Alexander B. kann die Situation zügig abgearbeitet werden: Die körperlichen Befunde werden mittels der verschlüsselten VideokonferenzSoftware an den Telemediziner in der Universitätsklinik für Anästhesiologie im Klinikum Oldenburg übertragen. Da die Schmerzen ausstrahlen, wird zur Sicherheit ein 12-Kanal-EKG übertragen und vom Arzt befundet.

Schnell sind sich Notfallsanitäter und Arzt einig: Es herrscht keine akute Gefahr. Wahrscheinlich war die lange Gartenarbeit von Alexander B. an den Rückenschmerzen schuld. Die im Algorithmus vorgesehene orale Analgesie wird ausgegeben und Alexander B. aufgefordert, sich weiterhin aktiv zu bewegen und ggf. zusätzlich Wärme zu applizieren. Sollten die Schmerzen nach dem Wochenende weiter bestehen, soll er sich beim Hausarzt vorstellen. Bei akuter Verschlechterung stehen KV-Bereitschaftsdienst oder Rettungsdienst für eine Wiedervorstellung zur Verfügung.

Johannes D. setzt seine Fahrt zum nächsten Patienten fort. Alexander B. kann nach einer halben Stunde dank der Wirkung der Tabletten ohne weitergehende Probleme schmerzfrei das Wochenende verbringen.

So ist das Modellprojekt konform der Strukturempfehlung für prähospitale Telemedizin der DGAI [10]. Rechtlich bleibt die Verantwortung für Diagnose und Behandlung des/der Patienten/-in beim Einsatz des Telemediziners bzw. der Telemedizinerin unverändert. Die Gesamtverantwortung für Diagnose und Behandlungsanweisungen trägt der Arzt bzw. die Ärztin, die Verantwortung für die adäquate Durchführung der/die ausführende Notfallsanitä- ter/-in. Der Einsatz von Telemedizin ändert somit nichts am bisherigen Verantwortungs- oder Haftungsgefüge.

Dass ein solcher Einsatz von Telemedizin möglich ist, verdanken die Patienten/-innen einer Stellungnahme der Bundesärztekammer 2015 [8]. Darin wurde in verschiedenen Modellen klargelegt, wann das sogenannte Fernbehandlungsverbot nicht greift. Telemedizin zwischen Arzt/Ärztin und examiniertem/-r Notfallsanitäter/-in präklinisch wurde damit zum ersten Mal juristisch sauber möglich.

Inzwischen steht die Umsetzung eines Beschlusses des 121. Ärztetags 2018 zur Novellierung der Musterberufsordnung an. Alle Landesärztekammern sind nun gefragt, in ihren Berufsordnungen auch ausschließliche Fernbehandlung unter Wahrung der ärztlichen Sorgfaltspflicht zuzulassen. Etliche Landesärztekammern haben dies bereits in Landesrecht umgesetzt. Spannend wird es, inwieweit sich landesspezifische Unterschiede ergeben werden.

\section{Rückmeldungen aus der Bevölkerung}

Seit dem 01.07.2018 sind an jedem Wochenende die Einsatzkräfte im Notdienstbezirk Delmenhorst unterwegs. Im zweiten Halbjahr 2018 hatten sie 143 Einsätze. Bei 97 Einsätzen wurde der/die Telemediziner/-in mit eingeschaltet. Die ersten Rückmeldungen aus der Bevölkerung sind durchweg positiv. Dass der Arzt bzw. die Ärztin nicht körperlich anwesend ist, sondern sich eine Fachkraft vor Ort kümmert und den Arzt bzw. die Ärztin bei Bedarf zuschaltet, wurde bisher in keinem Fall moniert.

\section{KERNAUSSAGEN}

- Engpässe in der medizinischen Versorgung sind in manchen Regionen besonders markant.

- Im niedersächsischen Modellprojekt 116117 füllen Gesundheitsfachkräfte die Lücke im KV-Notdienst. Die speziell geschulten Notfallsanitäter/innen suchen die Patienten/-innen zu Hause auf.

- Bei Bedarf schalten sie per Telemedizin einen Klinikarzt bzw. eine Klinikärztin hinzu. 12-KanalEKG, Pulsoxymetrie und Blutdruck können via Live-Schaltung übertragen werden.

- Das Projekt läuft seit 1.7.2018. Die Rückmeldungen aus der Bevölkerung sind bislang durchweg positiv.

\section{Interessenkonflikt}

Die Autoren geben an, dass kein Interessenkonflikt besteht. 
Autorinnen/Autoren

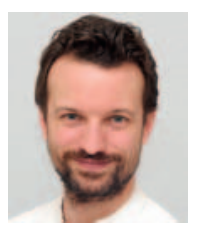

\section{Daniel Overheu}

Dr. med., D.E.S.A., Studium der Humanmedizin 2000 bis 2006 an der Med. Hochschule Hannover, Dissertation zum Dr. med. 2007, Facharztausbildung Anästhesiologie 2007 bis 2014 in Bad Zwischenahn, Westerstede, Leer und Oldenburg, Ärztlicher Leiter Telemedizin seit 2016 und Oberarzt im Team Kardioanästhesie seit 2017 an der Universitätsklinik für Anästhesiologie, Klinikum Oldenburg, Notarzt, LNA, spezielle Intensivmedizin.

Korrespondenzadresse

Dr. Daniel Overheu

Klinikum Oldenburg AöR

Rahel-Straus-Str. 10

26133 Oldenburg

overheu.daniel@klinikum-oldenburg.de

\section{Literatur}

[1] Statistisches Bundesamt. 13. koordinierte Bevölkerungsvorausberechnung. Wiesbaden, 2015

[2] Statistisches Bundesamt. Hohe Kosten im Gesundheitswesen: Eine Frage des Alters? Wirtschaft und Statistik Juli 2011

[3] Bundesärztekammer. Ärztestatistik. 2016

[4] Statistisches Bundesamt. Anzahl der Studienanfänger/-innen im ersten Hochschulsemester im Fach Humanmedizin (Allgemein-Medizin) in Deutschland vom Wintersemester 1998/1999 bis $2016 / 2017$.
[5] Kassenärztliche Bundesvereinigung. Bedarf an Hausärzten nimmt zu. KBV Praxisnachrichten, 27.4.2017.

[6] Entwicklung der Anzahl von Hausärzten in Deutschland in den Jahren 2005 bis 2020. Im Internet: https://de.statista.com/sta tistik/kategorien/kategorie/9/themen/74/branche/aerztekrankenhaeuser-apotheken/. Stand: 19.03.2019

[7] Niedersächsisches Ministerium für Landwirtschaft und Verbraucherschutz. Steuereinnahmekraft der kreisfreien Städte und Landkreisbereiche in den übrigen Regionen Niedersachsens im Durchschnitt der Jahre 2013-2015. Im Internet: https://www.google.de/url?sa=t\&rct=j\&q=\&esrc=s\&sour ce=web\&cd=1\&ved=2ahUKEwiugeTOjKDiAhVDZ1AK HYV1AK8QFjAAegQIBRAC\&url=https\%3A\%2F\%2Fwww.statis tik.niedersachsen.de\%2Fdownload\%2F120675\&usg=AOvVaw 012PJwkHxZGI4o-NilrCzL. Stand: 19.03.2019

[8] Hinweise und Erläuterungen zu § 7 Absatz 4 MBO-Ä (Fernbehandlung).

[9] Bundesärztekammer. Protokoll zum 113. Deutschen Ärztetag zum Antrag des Vorstandes und der Drucksache V-03a. Im Internet: www.bundesaerztekammer.de/aerztetag/be schlussprotokolle-ab-1996/113-daet-2010/top-v/telemedi zin/1-voraussetzungen/; Stand: 19.03.2019

[10] Kommission für Telemedizin der Deutschen Gesellschaft für Anästhesiologie und Intensivmedizin e.V. Telemedizin in der prähospitalen Notfallmedizin: Strukturempfehlung der DGAI. Im Internet: www.bda.de/files/Mrz_2016_-_Aus_der_Kom mission_Telemedizin.pdf; Stand: 19.03.2019

\section{Bibliografie}

DOI https://doi.org/10.1055/a-0886-8017

retten 2019; 8: 166-170

(c) Georg Thieme Verlag KG, Stuttgart · New York ISSN 2193-2387 